\title{
Correlation of high-sensitivity C-reactive protein and carotid plaques with coronary artery disease in elderly patients
}

\author{
YANHONG LIANG, YUANPING HOU, HONGYU NIU, MEI LU, LEI XUE and QIANMEI SUN \\ Department of Geriatric Medicine, Beijing Chaoyang Hospital, Capital Medical University, Beijing 100020, P.R. China
}

Received August 2, 2014; Accepted April 13, 2015

DOI: $10.3892 /$ etm.2015.2486

\begin{abstract}
The aim of this study was to explore the correlation of high-sensitivity C-reactive protein (hs-CRP) and carotid plaques with the severity of coronary artery disease (CAD). A total of 140 patients with angina who underwent coronary angiography were selected and divided into a single-vessel disease group $(n=11)$, double-vessel disease group $(n=18)$, multi-vessel disease group $(n=71)$ and control group $(n=40)$. Color Doppler ultrasound was applied to measure the intima-media thickness (IMT) of the common carotid artery (CCA) and carotid bifurcation, and to record the location and number of carotid plaques. The serum hs-CRP level was also determined. Compared with the control and single-vessel disease groups, the multi-vessel disease group showed significantly higher IMT of the CCA $(\mathrm{P}<0.05)$. The serum hs-CRP level was highest in the multi-vessel disease group $(\mathrm{P}<0.01)$. The hs-CRP level and carotid plaque characteristics were found to correlate closely with the severity of CAD. Therefore, they may be used to improve the prediction of CAD severity in elderly patients.
\end{abstract}

\section{Introduction}

Atherosclerosis is a chronic inflammatory disease (1) involving the cerebral artery, carotid artery, aorta, coronary artery, renal artery and arteries of the lower extremities, which is most common in the tunica intima and media of large and medium-sized arteries. Inflammation can cause unstable plaques to rupture and induce thrombosis, leading to subsequent acute ischemic events; an increase in the intima-media thickness (IMT) of an artery is a marker of systemic and coronary atherosclerosis (2). Coronary artery disease (CAD) also known as atherosclerotic heart disease, is

Correspondence to: Dr Qianmei Sun, Department of Geriatric Medicine, Beijing Chaoyang Hospital, Capital Medical University, 8 Gong-Ti South Road, Beijing 100020, P.R. China

E-mail: qianmeisun@163.com

Key words: elderly patients, high-sensitivity C-reactive protein, carotid plaque, coronary artery disease characterized by abnormal lipid metabolism, the deposition of lipids in the arterial intima and plaque formation, which results in arterial stenosis, blood flow obstruction and cardiac ischemia (3).

Numerous researchers have discovered that atherosclerotic plaque involves not only lipid deposition, but also occult chronic inflammation in systemic arteries, as described in a previous review (4). Inflammatory cytokines are produced and released during coronary stenosis, which, in turn, may further promote coronary stenosis. C-reactive protein (CRP), an acute-phase protein that arises rapidly and reaches high levels in plasma in response to infection or tissue injury, plays an important role in the occurrence and progress of inflammation; hence, it has attracted considerable attention. CRP activates the complement system and enhances phagocytosis to remove pathogens and damaged, necrotic or apoptotic cells $(5,6)$. Studies have also demonstrated that CRP is an independent predictor of carotid IMT $(7,8)$. High-sensitivity CRP (hs-CRP) is a nonspecific acute-phase protein that has been recognized to be an independent predictor of cardiovascular events $(9,10)$, and is thus of particular interest

CAD is a common disease that is a major threat to middle-aged and elderly individuals worldwide. Atherosclerotic changes in superficial carotid arteries and their severity generally reflect systemic atherosclerosis; they are easily detected with high-frequency ultrasound in the early stage of the disease. An artery with an elevated IMT is a marker of systemic and coronary atherosclerosis (2). As a significant factor in the inflammatory process, hs-CRP is a very sensitive indicator of the development of atherosclerosis, which is useful in the diagnosis of CAD and for risk stratification (11). In the present study, hs-CRP levels and carotid plaque characteristics were detected and their correlation with the severity of CAD was explored.

\section{Materials and methods}

Subjects. A total of 140 elderly patients ( $\geq 60$ years) in accordance with WHO diagnostic standards for angina, myocardial infarction or chest pain (12) were selected according to clinical manifestations and coronary angiography from Beijing Chaoyang Hospital (Beijing, China) between January 2010 and December 2012. The subjects, comprising 89 males and 51 females aged 60-85 years with a mean age of $70.43 \pm 5.95$ years, were divided into a single-vessel disease group $(n=11)$, double-vessel disease 
group ( $\mathrm{n}=18)$, multi-vessel disease group $(\mathrm{n}=71)$ and control group $(n=40)$. The patients in the control group were 23 males and 17 females that were without stenosis or did not meet the full diagnostic criteria for stenosis. All the subjects were of Han origin without any blood relationship. There were no statistical differences in age, gender, blood glucose levels, lipid levels and smoking history among the groups. Exclusion criteria: Patients complicated by organic heart diseases, such as myocarditis and myocardiopathy, liver and kidney dysfunction, hematologic diseases, connective tissue disease, tumor and infectious diseases. The subjects had no history of thromboembolic disease and had not used anticoagulants recently. This study was conducted in accordance with the Declaration of Helsinki and with approval from the Ethics Committee of Capital Medical University. Written informed consent was obtained from all participants.

Coronary angiography. Selective coronary angiography was performed using the standard Judkins technique (13) in multiple positions. The diagnostic criterion was stenosis $\geq 50 \%$ in $\geq 1$ coronary vessel.

Detection of hs-CRP level. Fasting venous blood was sampled from all the patients on the first morning following admission and the serum was isolated for further analysis. The hs-CRP level was detected by hs-CRP microparticle-enhanced nephelometric immunoassay using a kit purchased from Orion Diagnostica (Espoo, Finland). The expected value for healthy individuals is $<3 \mathrm{mg} / \mathrm{l}$.

Carotid ultrasound. Color Doppler ultrasound was performed with a probe frequency of $7.5 \mathrm{MHz}$, using an Aplio 500 ultrasound device (Toshiba Corporation, Tokyo, Japan). The patient was examined in the supine position with his/her head turned contralaterally by $45^{\circ}$. A long axial section of the carotid artery was probed to show the common carotid artery (CCA) bifurcation and the internal carotid artery. The origin, main part and bifurcation of the CCA were examined on both sides to investigate the blood vessel morphology and endarterium, and to observe any atherosclerosis plaques. IMT is defined as the perpendicular distance between the main surface of the intima and the interface of the tunica media and adventitia. IMT at the carotid bifurcation and $1.5 \mathrm{~cm}$ proximal to the bifurcation was measured and the maximum value was recorded. An IMT $>1.0 \mathrm{~mm}$ was considered as thickening and IMT $\geq 1.2 \mathrm{~mm}$ was labeled as atherosclerotic plaque. Whether the carotid intima was smooth, and the location, amount and properties of the atherosclerotic plaques were observed.

Carotid plaque scoring. The patients were semi-quantitatively scored on a scale of 0-4 as follows: Score 0 , no plaque; score 1 , only 1 plaque with a thickness $<2 \mathrm{~mm}$; score 2,2 plaques with a thickness $<2 \mathrm{~mm}$ or only 1 plaque with a thickness $>2 \mathrm{~mm}$; score 3,2 plaques at least one of which had a thickness $<2 \mathrm{~mm}$; score 4, 2 plaques with thickness $>2 \mathrm{~mm}$ or $>50 \%$ carotid stenosis (14).

Statistical analysis. Data was analyzed with SPSS software version 19.0 (SPSS, Inc., Chicago, IL, USA). Measurement data are presented as the mean \pm standard error of the mean.
Table I. Correlation between serum hs-CRP level and CAD.

\begin{tabular}{lcc}
\hline Group & Cases & Hs-CRP (mg/l) \\
\hline Control & 40 & $1.64 \pm 0.98$ \\
Single-vessel disease & 11 & $2.57 \pm 1.18$ \\
Double-vessel disease & 18 & $5.22 \pm 3.48^{\mathrm{a}}$ \\
Multi-vessel disease & 71 & $7.46 \pm 3.63^{\mathrm{b}}$ \\
\hline
\end{tabular}

Hs-CRP levels are presented as mean \pm standard error of the mean. ${ }^{\mathrm{a}} \mathrm{P}<0.05$ vs. single-vessel disease group. ${ }^{\mathrm{b}} \mathrm{P}<0.01$ vs. single-vessel disease and double-vessel disease groups. Hs-CRP, high-sensitivity C-reactive protein; CAD, coronary artery disease.

Comparison of IMT and plaque scores between groups was conducted by analysis of variance and Student's $t$-test. The correlation of vascular disease with IMT and plaque score employed single factor analysis. $\mathrm{P}<0.05$ was considered to indicate a statistically significant difference.

\section{Results}

Correlation between serum hs-CRP level and CAD. The serum level of hs-CRP in the double-vessel disease group was higher than that of the single-vessel disease group $(\mathrm{P}<0.05)$, and the highest hs-CRP level was observed in the multi-vessel disease group $(\mathrm{P}<0.01)$. The serum level of hs-CRP was found to increase as the number of vessels involved in CAD increased (Table I).

Comparison of CCA-IMT, carotid bifurcation IMT and plaque score. The double-vessel disease group exhibited a higher carotid bifurcation IMT and plaque score than the single-vessel disease and control groups. These variables, together with the CCA-IMT were highest in the multi-vessel disease group (Table II).

Comparison of the proportion of cases with increased CCA-IMT and carotid bifurcation IMT. The proportion of cases with increased carotid bifurcation IMT was significantly higher in the single-, double- and multi-vessel disease groups than in the control group $(\mathrm{P}<0.05)$. Compared with the single-vessel disease group, the multi-vessel disease group showed a distinctly higher proportion of cases of increased CCA-IMT ( $\mathrm{P}<0.05$; Table III). The proportion of cases with increased CCA-IMT and carotid bifurcation IMT was found to increase as the number of vessels involved in CAD increased.

\section{Discussion}

The pathogenesis of coronary atherosclerosis is complex, involving endothelial cell injury, lipid infiltration and the conversion of macrophages into lipid-laden foam cells, which closely correlates with inflammation (15). The inflammatory response is of importance in the occurrence, development and progression of CAD. Serum levels of inflammatory markers can reflect disease severity and may be used to predict acute coronary events (16). 
Table II. Comparison of CCA-IMT, carotid bifurcation IMT and plaque score among groups.

\begin{tabular}{lcccc}
\hline Group & Cases & CCA-IMT & Carotid bifurcation IMT & Plaque score \\
\hline Control & 40 & $0.83 \pm 0.15$ & $1.03 \pm 0.22$ & $2.12 \pm 1.91$ \\
Single-vessel disease & 11 & $0.97 \pm 0.26$ & $1.12 \pm 0.14$ & $2.98 \pm 2.02$ \\
Double-vessel disease & 18 & $1.02 \pm 0.17$ & $1.23 \pm 0.35^{\mathrm{b}}$ & $3.62 \pm 2.24^{\mathrm{b}}$ \\
Multi-vessel disease & 71 & $1.26 \pm 0.21^{\mathrm{a}}$ & $1.42 \pm 0.40^{\mathrm{b}}$ & $4.28 \pm 2.30^{\mathrm{b}}$ \\
\hline
\end{tabular}

Values are presented as mean \pm standard error of the mean. ${ }^{\mathrm{a}} \mathrm{P}<0.05,{ }^{\mathrm{b}} \mathrm{P}<0.01 \mathrm{vs}$. control group. CCA, common carotid artery; IMT, intima-media thickness.

Table III. Proportion of cases with increased CCA-IMT and carotid bifurcation IMT.

\begin{tabular}{lccc}
\hline Group & No. of cases & Increased CCA-IMT (\%) & Increased carotid bifurcation IMT (\%) \\
\hline Control & 40 & 7.5 & 45.0 \\
Single-vessel disease & 11 & 18.2 & $72.7^{\mathrm{a}}$ \\
Double-vessel disease & 18 & 22.2 & $83.3^{\mathrm{a}}$ \\
Multi-vessel disease & 71 & $45.1^{\mathrm{a}, \mathrm{b}}$ & $87.3^{\mathrm{a}}$
\end{tabular}

${ }^{\mathrm{a}} \mathrm{P}<0.05$ vs. control group; ${ }^{\mathrm{b}}<0.05$ vs. single-vessel disease group. CCA, common carotid artery; IMT, intima-media thickness.

In the present study, it was found that the serum hs-CRP level was associated with CAD severity, as higher serum levels of hs-CRP were observed in patients with double- and multi-vessel disease. Previous research (17) has shown that hs-CRP promotes plaque formation and that the CRP level in peripheral blood parallels the inflammation severity in plaques. Chambless et al (18) have demonstrated that the incidence of carotid atherosclerosis and plaques increases with increasing CRP level, which positively correlates with plaque score and carotid IMT. CRP may serve as a pathogenic factor in the occurrence and development of atherosclerosis (19). The serum level of hs-CRP increases as the severity and extent of CAD increases, indicating that hs-CRP is associated with CAD occurrence and progress, and thus can serve as a predictor of CAD severity and cardiovascular events.

Atherosclerosis is a systemic disease that mainly affects large- and medium-sized arteries and simultaneously affects carotid and coronary arteries. The severity of a carotid plaque can reflect the severity of CAD to a certain extent. A large number of studies have shown that carotid IMT is an independent risk factor for coronary stenosis, carotid atherosclerosis occurs in parallel with coronary atherosclerosis and CAD severity significantly increases with elevated IMT $(2,3,17,18)$.

In the present study, higher CCA-IMT and a greater number of cases of carotid bifurcation IMT were observed in patients with CAD than in controls, particularly in the multi-vessel disease group, which is consistent with previous findings (20). This suggests that early carotid ultrasound examination in patients with risk factors may be used to facilitate the diagnosis of CAD severity. In the process of atherosclerosis, the endarterium is involved initially and elevated IMT is a marker of early atherosclerosis (21). The higher the IMT in patients with CAD the higher the incidence of adverse cardiac events. ELSA research has demonstrated that IMT measurements in asymptomatic middle-aged or elderly patients can provide extra information to facilitate the risk stratification of CAD in addition to conventional risk factors (22).

Carotid IMT has been recognized as an alternative marker of atherosclerosis for the evaluation of the severity of systemic atherosclerosis and directly correlates with the risk of vascular disease. Elevated carotid IMT may be part of arterial remodeling in early atherosclerosis, while carotid plaques may represent late atherosclerosis induced by inflammation, oxidation, endothelial dysfunction and smooth muscle cell proliferation; thus, plaques are superior to IMT in reflecting the impact of human internal factors (22). The present study demonstrated that CCA and carotid bifurcation IMT closely correlate with CAD severity. Therefore, carotid IMT and plaque score can be used for the clinical diagnosis of CAD.

A rapid increase in the number of elderly individuals has occurred as the population ages. CAD is prevalent in elderly people; however, some elderly patients refuse to undergo coronary angiography to determine CAD severity because of age, social and family factors. Measurements of hs-CRP levels and carotid ultrasound examinations are practical, valuable, painless, noninvasive, quantitative, safe, simple, reproducible and easy to perform, which promotes their application in clinical and scientific research. If subclinical atherosclerosis, that is, elevated IMT, is observed, aggressive treatment and the identification of patients without CAD should be performed to determine what further drug therapy is required and to reduce cardiovascular events. 


\section{References}

1. Ross R: Atheroscherosis - an inflammatory disease. N Eng J Med 340: 115-126, 1999.

2. Kablak-Ziembicka A, Przewlocki T, Pieniazek P, et al: The role of carotid intima-media thickness assessment in cardiovascular risk evaluation in patients with polyvascular atherosclerosis Atherosclerosis 209: 125-130, 2010.

3. Kabłak-Ziembicka A, Przewłocki T, Stępień E, et al: Relationship between carotid intima-media thickness, cytokines, atherosclerosis extent and a two-year cardiovascular risk in patients with arteriosclerosis. Kardiol Pol 69: 1024-1031, 2011.

4. Rifai N and Ridker PM: High-sensitivity C-reactive protein A novel and promising marker of coronary heart disease. Clin Chem 47: 403-411, 2001.

5. Bokhari SA, Khan AA, Butt AK, et al: Non-surgical periodontal therapy reduces coronary heart disease risk markers: A randomized controlled trial. J Clin Periodontol 39: 1065-1074, 2012.

6. Cook NR: The case for C-reactive protein as a risk marker for coronary heart disease. Ann Intern Med 152: 406-407, 2010

7. Toprak A, Kandavar R, Toprak D, et al: C-reactive protein is an independent predictor for carotid artery intima-media thickness progression in asymptomatic younger adults (from the Bogalusa Heart Study). BMC Cardiovasc Disord 11: 78, 2011.

8. Molino-Lova R, Macchi C, Gori AM, et al: High sensitivity $\mathrm{C}$-reactive protein predicts the development of new carotid artery plaques in older persons. Nutr Metab Cardiovasc Dis 21 776-782, 2011

9. Joshi MS, Tong L, Cook AC, et al: Increased myocardial prevalence of C-reactive protein in human coronary heart disease: Direct effects on microvessel density and endothelial cell survival. Cardiovasc Pathol 21: 428-435, 2012.

10. Ko YG, Le VC, Kim BH, et al: Correlations between coronary plaque tissue composition assessed by virtual histology and blood levels of biomarkers for coronary artery disease. Yonsei Med J 53: 508-516, 2012.

11. Schlager O, Exner M, Mlekusch W, et al: C-reactive protein predicts future cardiovascular events in patients with carotid stenosis. Stroke 38: 1263-1268, 2007.
12. Hannan EL, Samadashvili Z, Cozzens K, et al: Assessment of the new appropriate use criteria for diagnostic catheterization in the detection of coronary artery disease following noninvasive stress testing. Int J Cardiol 170: 371-375, 2014.

13. Guillard N, Lefèvre T, Spaulding C, et al: Coronary angiography by left radial approach: A bi-center prospective pilot study. Arch Mal Coeur Vaiss 90: 1349-1355, 1997 (In French).

14. Zureik M, Bureau JM, Temmar M, et al: Echogenic carotid plaques are associated with aortic arterial stiffness in subjects with subclinical carotid atherosclerosis. Hypertension 41: 519-527, 2003.

15. Libby P, Ridker PM and Maseri A: Inflammation and atherosclerosis. Circulation 105: 1135-1143, 2002.

16. Blake GJ and Ridker PM: C-reactive protein and other inflammatory risk markers in acute coronary syndromes. J Am Coll Cardiol 41 (4 Suppl): 37S-42S, 2003.

17. Johnston SC, Zhang H, Messina LM, Lawton MT and Dean D: Chlamydia pneumoniae burden in carotid arteries is associated with upregulation of plaque interleukin-6 and elevated C-reactive protein in serum. Arterioscler Thromb Vasc Biol 25: 2648-2653, 2005.

18. Chambless LE, Folson AR, Cheqq LX, et al: Carotid wall thickness is predictive of incident clinical stroke: The Atherosclerosis Risk in Communities (ARIC) study. Am J Epidemiol 151: 478-487, 2000.

19. Heuten H, Goovaerts I, Ennekens G and Vrints C: Carotid artey intima-media thickness is associated with coronary artery disease. Acta Cardiol 63: 309-313, 2008.

20. Seo WK, Yong HS, Koh SB, et al: Correlation of coronary artery atherosclerosis with atherosclerosis of the intracranial cerebral artery and the extracranial carotid artery. Eur Neurol 59: 292-298, 2008.

21. Nguyen-Thanh HT and Benzaquen BS: Screening for subclinical coronary artery disease measuring carotid intima media thickness. Am J Cardiol 104: 1383-1388, 2009.

22. Zanchetti A, Hennig M, Hollweck R, et al: Baseline values but not treatment-induced changes in carotid intima-media thickness predict incident cardiovascular events in treated hypertensive patients: Findings in the European Lacidipine Study on Atherosclerosis (ELSA). Circulation 120: 1084-1090, 2009. 